\title{
Relation between a career and family life for English hospital consultants: qualitative, semistructured interview study
}

Carol Dumelow, Peter Littlejohns, Sîan Griffiths

\begin{abstract}
Objective To explore the relation between work and family life among hospital consultants and their attitude towards the choices and constraints that influence this relation.

Design Qualitative study of consultants' experiences and views based on tape recorded semistructured interviews.

Setting Former South Thames health region in southeast England.

Participants 202 male and female NHS hospital consultants aged between 40 to 50 years representing all hospital medical specialties.

Results Three types of relation between work and family life (career dominant, segregated, and accommodating) were identified among hospital consultants. Most consultants had a segregated relation, although female consultants were more likely than male consultants to have a career dominant or an accommodating relation. Many male consultants and some female consultants expressed considerable dissatisfaction with the balance between their career and family life. A factor influencing this dissatisfaction was the perceived lack of choice to spend time on their personal or family life, because of the working practices and attitudes within hospital culture, if they wanted a successful career.

Conclusions Consultants are currently fitting in with the profession rather than the profession adapting to enable doctors to have fulfilling professional and personal lives. Current government policies to increase the medical workforce and promote family friendly policies in the NHS ought to take account of the need for a fundamental change in hospital culture to enable doctors to be more involved in their personal or family life without detriment to their career progress.
\end{abstract}

\section{Introduction}

In the past, doctors were expected to plan their professional and domestic lives in line with William Osler's view of a medical career ("heavy as are your responsibilities to those nearest and dearest, they are outweighed by the responsibilities to yourself, to the profession and to the public"1). Over a century later, Department of Health initiatives are directed at increasing the size of the medical workforce and promoting family friendly policies and retainer schemes. ${ }^{23}$ Organisational practices as factors in influencing the balance between work and family life have been debated in the social science literature. ${ }^{4-7}$ In the medical literature, considerable attention has been given to the way women doctors balance their work and family commitments. ${ }^{8-13}$ With three exceptions, ${ }^{14-16}$ studies have focused on women doctors, and little research has been conducted among hospital consultants. Up to date information is needed if family friendly policies are to be adopted. We interviewed consultants about the relation between their work and their personal or family life and how they feel about the choices and constraints influencing this relation.

\section{Methods}

Participants and setting

Disproportionate stratified sampling was used to obtain a sample of 286 male and female hospital consultants aged 40-50 years working in all medical specialties in NHS hospitals in the former South Thames health region in southeast England. We estimated that this sample would provide data on 100 male and 100 female consultants (based on a 70\% response rate). Hospital specialties were grouped according to three criteria: the hours worked; the type of work undertaken; and the amount of on-call work required. A sampling fraction of 1 in 2 women and 1 in 6 men was used for the diagnostic, medical, psychiatry, and anaesthetics specialties. For the surgical specialty group, all women were selected, and a sampling fraction of 1 in 9 men was used. For the obstetrics and gynaecology group, all women were selected and a 1 in 6 sample was taken for the men.

\section{Interviews}

We designed a semistructured interview schedule on the basis of key themes identified from analysis of in-depth interviews with five doctors; discussions with senior academics involved in research studies of hospital doctors; the research questions for the study; and issues prevalent in a review of the medical and sociological literature. The schedule was piloted with a sample of hospital consultants, and changes were made. The final schedule included open ended and precoded questions on five topics (box). Consent to participation in the study was voluntary and was gained from consultants by letter and follow up telephone call. Most consultants agreed to participate. Interviews varied considerably in length, ranging from 45 to 90 minutes (average length one hour); the variation seemed to be associated with different personality types. Most consultants talked candidly about their personal lives, some were grateful for the opportunity to express concerns.

Interviews were conducted in the consultant's workplace or home. Interviews were conducted by one of the authors (CD) and four social scientists, all of whom had experience in interviewing skills and had received additional training for the study. The interviews were audiotaped to allow assessment of interviewers' reliability and to enable the answers to the open ended questions to be transcribed verbatim. Curricula vitae were obtained from the consultants before

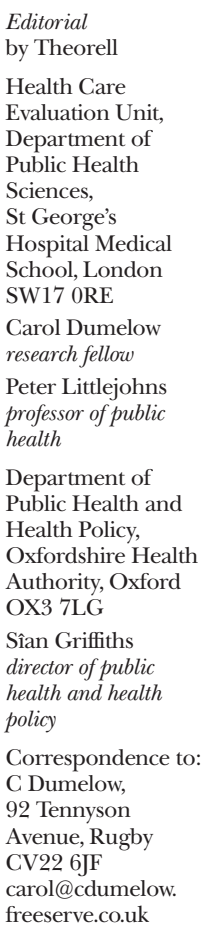

BMJ 2000;320:1437-40 


\section{Topics covered in interviews}

Work history

- Employment status, past and current

- Reasons for employment history

- Reasons for choice of specialty

Domestic life

- Marital status

- Partner's support for participant's career

- Children and childcare responsibilities

- Management of child care

- Management of household tasks

- Effect of personal and family life on career

- Effect of medical career on personal and family life

Postgraduate medical training

- Geographical mobility

- Effect of organisational working practices on life outside medicine

- Career structure and effect on personal and family life

Medical environment during training

- Perceptions of attitudes towards women doctors

- Perceptions of medical culture

Achievement

- Attitude towards own achievement in medical career

- Career aspirations for the future

interview, and these were used to collect information on work history.

\section{Analysis}

The transcribed answers to the open ended questions were analysed using the "framework" qualitative data analysis method. ${ }^{17}$ The transcripts were studied carefully, and recurring themes were noted. A coding framework was developed from the recurring themes and the aims of the research. This framework was applied systematically to the transcripts to enable the interview data to be coded according to the framework categories. Category codes for each consultant were transferred to a framework chart so that the reorganised data could be analysed. Patterns and differences were identified, and three types of relation between work and family were created (career dominant, segregated, and accommodating). The criteria defining these types were based on aspects of their personal or family life or of their career that had been adjusted or modified in some way to benefit career or personal or family life (box). One of the interviewers (Suzanne Cohen), one of the authors (PL), and another social scientist (Sara Arber) helped the validation process by discussing the development of the categories and the creation of the typologies (and the associations made), which were confirmed or modified as necessary. Quotes used in this paper were selected to represent the range of responses.

\section{Results}

Characteristics of consultants interviewed

After exclusion of 11 consultants from the sample of 286 because they were not aged $40-50$ years, were no longer working in the South Thames region, or had left hospital medicine, 202 consultants were interviewed ( $73 \%$ response rate). The table ${ }^{1}$ shows the characteristics of respondents. The proportion of women who responded $(82 \%)$ was higher than the proportion of men who responded $(64 \%)$; smaller proportions of men than women in the surgical $(58 \% v 94 \%)$ and diagnostic (53\% v 93\%) specialties responded; and a smaller proportion of women than men in anaesthetics $(67 \%$ v 74\%) responded. A fifth of respondents were consultants in teaching hospitals. Most $(82 \%)$ of the consultants interviewed were married, and most $(79 \%)$ respondents had children. Of consultants with children, about a third had children aged $\leq 5$ years. As in other studies, ${ }^{15}{ }^{16}$ women were more likely than men to be younger, single, and childless. Women were also more likely than men to have married at a later grade in their career $(62 \% v 54 \%)$; women who had children were more likely to have a child aged $\leq 5$ years $(37 \%)$; and women were more likely to have achieved consultant grade at a younger age (table).

\section{Work and family relations}

Relations between work and family life are by nature fluid and dynamic. It is difficult to distinguish between cause and effect, and we have not tried to identify causal relations in this paper. Instead, we examine some of the factors that consultants considered important in shaping the balance between their work and their personal or family life (see previous box on typologies).

\section{Career dominant relation}

About a fifth of women and three men believed that their family or personal life had been restricted during

Criteria used to develop typologies for work and family relations, and characteristics of consultants in these typologies

Criteria

- Modifications made to personal and family life to benefit medical career

- Modifications made to career for perceived benefit to personal and family life

\section{Characteristics}

Career dominant relation between work and family

- Single and childless

- Divorced and childless

- Full time continuous career

Segregated relation between work and family

- Married or cohabiting with children

- Married or cohabiting without children

- Divorced with children and primary carer

- Full time continuous career

- Family responsibilities organised to enable more time to be involved in career

Accommodating relation between work and family

- Married or cohabiting with children

- Men: full time continuous career and career goals or work commitments reduced to be more involved in family life

- Women: Career break or periods of less than full time training or work (or both of these) because of family responsibilities 
their 20 s and early 30 s, either intentionally or unintentionally, for the benefit of their career. Two women had made a conscious choice at an early stage in their career to forgo having children. The remaining consultants in this group, however, had wanted to develop relationships and have children but thought that the constraints of working practices in hospital medicine and perceived pressure to follow a full time career had been influential in shaping the balance between their career and their personal or family life. Some consultants expressed strong regrets at giving so much time to their medical career. (See box for examples of respondents' comments.)

\section{Segregated relation}

Two thirds of men and just over half of women had highly segregated professional and family lives. Family responsibilities were organised to enable more time to be devoted to their medical career. For both men and women this involved full time domestic support-from a spouse or partner or from a paid domestic worker, or from both. Most women in this group thought that this approach was the only way they could achieve consultant grade and have a family, and most were satisfied with the balance between their career and family life, when set in the context of their perceived choices and constraints. Many men in this group, however, were dissatisfied with the balance between their career and family life. This was due largely to the perceived lack of choice to spend time in their family life (because of working practices and pressure to conform to these practices) if they were to have a successful medical career. (See box for respondents' comments.)

\section{Accommodating relation}

About a tenth of men and a third of women had an accommodating relation between work and family. All women in this group had worked part time or taken a career break at some point in their career. Men had restricted their work commitments or limited their career goals to benefit their personal or family life. Most men and women in this group expressed satisfaction with the balance between their career and family life. (See box for respondents' comments.)

\section{Comments from consultants with career dominant relation between work and family life}

"It may be that I haven't quite got the balance right because I'm aware I gave too much to medicine and lost out as a result." (Divorced, female, childless; obstetrics and gynaecology)

"I wouldn't start my medical career again. It's taken too much out of me. You don't have a life outside medicine, where other priorities seem not to weigh up. There is no pot of gold at the end of the rainbow. There are other ways of living too." (Single, female, childless; surgery)

"The long hours are very damaging; you tend to fall into a routine of work, eat, sleep, and you become alienated. The rewards to an individual can be tremendous but at great personal sacrifice in terms of life outside medicine." (Single, male, childless; diagnostics)
Characteristics of 202 male and female consultants interviewed about balance between work and family life. Values are numbers (percentages) of respondents

\begin{tabular}{|c|c|c|c|}
\hline & Male & Female & Total \\
\hline \multicolumn{4}{|l|}{ Age (years): } \\
\hline $40-45$ & $42(49)$ & $78(67)$ & $120(59)$ \\
\hline $46-50^{\star}$ & $44(51)$ & $38(33)$ & $82(41)$ \\
\hline All & $86(100)$ & $116(100)$ & $202(100)$ \\
\hline \multicolumn{4}{|l|}{ Specialty: } \\
\hline Diagnostic & $10(12)$ & $29(25)$ & $39(19)$ \\
\hline Medical & $28(32)$ & $31(27)$ & $59(29)$ \\
\hline Surgical & $15(17)$ & 17 (14) & $32(16)$ \\
\hline Psychiatry & $13(15)$ & $20(17)$ & $33(16)$ \\
\hline Obstetrics and gynaecology & $6(7)$ & $9(8)$ & $15(7)$ \\
\hline Anaesthetics & $14(16)$ & $10(9)$ & $24(12)$ \\
\hline \multicolumn{4}{|l|}{ Current employment status: } \\
\hline Full time (includes maximum part time) & $86(100)$ & $94(81)$ & $180(89)$ \\
\hline Less than full time & 0 & $22(19)$ & $22(11)$ \\
\hline \multicolumn{4}{|l|}{ Mean age at which consultant post achieved: } \\
\hline Diagnostic & 37.0 & 34.4 & 34.4 \\
\hline Medical & 37.1 & 37.2 & 37.2 \\
\hline Surgical & 37.2 & 36.6 & 36.6 \\
\hline Psychiatry & 38.0 & 37.2 & 37.2 \\
\hline Obstetrics and gynaecology & 40.7 & 37.0 & 37.0 \\
\hline Anaesthetics & 35.7 & 36.2 & 36.2 \\
\hline \multicolumn{4}{|l|}{ Marital status: } \\
\hline Single & $4(5)$ & $17(14)$ & $21(10)$ \\
\hline Married/cohabited (once) & $66(77)$ & $79(68)$ & $145(72)$ \\
\hline Married/cohabited (more than once) & $10(12)$ & $10(8)$ & $20(10)$ \\
\hline Divorced/separated/widowed & $6(7)$ & $10(9)$ & $16(8)$ \\
\hline \multicolumn{4}{|l|}{ Grade at first marriage: } \\
\hline Medical school & $10(13)$ & $13(15)$ & $23(14)$ \\
\hline General professional training & $25(33)$ & $21(24)$ & $46(28)$ \\
\hline Higher specialist training & $31(41)$ & $44(49)$ & $75(45)$ \\
\hline Consultant & $10(13)$ & $11(12)$ & $21(13)$ \\
\hline All & $76(100)$ & $89(100)$ & $165(100)$ \\
\hline \multicolumn{4}{|l|}{ Age (years) of youngest child: } \\
\hline No children & $10(12)$ & $32(28)$ & $42(21)$ \\
\hline $0-5$ & $17(20)$ & $31(27)$ & $48(24)$ \\
\hline $6-10$ & $27(31)$ & $27(23)$ & $54(27)$ \\
\hline $11-16$ & $25(29)$ & 17 (14) & $42(21)$ \\
\hline$\geqslant 17$ & $7(8)$ & $9(8)$ & $16(8)$ \\
\hline All & $86(100)$ & $116(100)$ & $202(100)$ \\
\hline \multicolumn{4}{|l|}{ Medical grade at birth of first child: } \\
\hline Student & $1(1)$ & $1(1)$ & $2(1)$ \\
\hline House officer & 1 (1) & 1 (1) & $2(1)$ \\
\hline Senior house officer & $10(13)$ & $13(16)$ & $23(15)$ \\
\hline Registrar & $25(33)$ & $15(18)$ & $40(25)$ \\
\hline Senior registrar & $22(29)$ & $23(28)$ & $45(29)$ \\
\hline Consultant & $16(21)$ & $29(35)$ & $45(29)$ \\
\hline All & $75(100)$ & $82(100)$ & $157 \dagger(100)$ \\
\hline \multicolumn{4}{|l|}{ Employment status of consultant's partner: } \\
\hline Full time/self employed & $24(31)$ & $75(84)$ & $99(60)$ \\
\hline Part time & $24(31)$ & $6(7)$ & $30(18)$ \\
\hline Unemployed/student/retired & $4(5)$ & $6(7)$ & $10(6)$ \\
\hline Housewife/househusband & $24(32)$ & 2 (2) & $26(16)$ \\
\hline All & $76(100)$ & $89(100)$ & $165(100)$ \\
\hline
\end{tabular}

*1 respondent was aged 51 at interview.

†Data not available in three cases.

\section{Discussion}

Despite reaching the pinnacle of a demanding professional career, many male consultants expressed reservations over its effect on their family and personal life. These reservations come from a generation who trained when medicine was more of a vocation and when limited personal and family life was perhaps more acceptable. Fewer reservations were expressed by the consultants (both male and female) who perceived 


\section{Comments from consultants with segregated relation between work and family life}

"I couldn't spread myself more thinly between work and home. I think I have got the level I wish to have as a consultant, which I can build on, and I've got a wonderful home life." (Married, female, children; surgery) "I'm not around as much as I would be if I had another career. My boys have grown up with the situation. If I have not been home I have rung. I would like to spend more time with them, but medicine takes everything except your soul." (Married, male, children; psychiatry)

"It is a pity that medicine in this country historically has been so badly organised that most clinicians in acute specialties work ridiculous hours, at the expense of their health and their families. But if you want to pursue a career in the acute specialties that's the way it is." (Married, male, children; surgery)

"It puts tremendous pressure on people. Doctors are put under pressure to spend a lot more time on medicine and hospital life than perhaps they ought to because of the way we are trained in medical school. It may mean you are exhausted and have nothing left for anyone else. Some weeks I am emotionally drained, exhausted." (Married, male, children; anaesthetics) increase the medical workforce and implement family friendly policies should take account of the need for a fundamental change in current hospital medical culture.

We thank all the consultants who took part in this study. We also thank Professor Sara Arber for her advice and supervision during the course of this study; social scientists Gillian Carey, Suzanne Cohen, Miranda Cormell, and Juliet Essen for their help with the interviews; and Dr Jane Harrison, Dr Jane Maher, and Professor Ramirez for their comments on the drafts of this paper.

Contributors: $\mathrm{CD}$ was the principal investigator. She initiated the research; discussed core ideas; and participated in the design and execution of the study and in the data collection, data documentation, quality control, analysis and interpretation of the data, and writing of the paper. PL initiated the research; discussed core ideas; and participated in the protocol design, analysis and interpretation of the data, and writing of the paper. SG initiated the research; discussed core ideas; participated in the protocol design; and edited the paper. $\mathrm{CD}$ is guarantor for the paper.

Funding: South Thames Regional Health Authority.

Competing interests: None declared.

they had a more balanced relation between their career and family life. Previous work has highlighted the changes needed in medicine. ${ }^{13}{ }^{15}$ Some changes have occurred ${ }^{18}{ }^{19}$ yet we found that the expectations placed on doctors to give more of their time to their medical career than is perhaps healthy for them or their family still prevail.

Currently doctors seem to be fitting in with the system rather than the system adapting to a changing workforce and enabling doctors to have fulfilling professional and personal lives. Government policies to

\section{Comments from consultants with accommodating relation between work and family life}

"To cope you need child care and communication, organisation and planning, and energy to enjoy it. You certainly need a lot of energy, and you've got to want both aspects of life. It's enhanced my life and I'm where I want to be." (Married, female, children; surgery)

“I didn't stay at work any longer than I had to. I was meant to go out for drinks after work with others including senior medical staff. I was meant to socialise with the team, but I didn't. As soon as my shift was finished, I was off home to my family. My family were important." (Married, male, children; diagnostics)
1 Verney RE. The student life-the philosophy of Sir William Osler. London: Livingstone, 1957.

Department of Health. Improving Working Lives. Wetherby: DoH, 1999.

White C. Mystery of "7000 extra doctors" BMJ 1998.317.300.

4 Itzin C, Newman J, eds. Gender, culture and organisational change. London: Routledge, 1995.

5 Lewis S. Family friendly employment policies: a route to changing organisational culture or playing about at the margins? Gender, Work and Organisation 1997;4(1):13-23.

6 Webb J. The politics of equal opportunity. Gender, Work and Organisation 1997;4:159-69.

7 Liff S, Cameron I. Changing equality cultures to move beyond women's problems. Gender, Work and Organisation 1997;4(1):35-46.

8 Swerdlow AJ, McNeilly RH, Rue R. Women doctors in training: problems and progress. BMJ 1980;281:754-8.

9 Ward AWM, Francis B, Dawson C, Ennis E, Morrison J. Careers of medical women. Sheffield: University of Sheffield, 1981.

10 Rhodes P. The career aspirations of women doctors who qualified in 1974 and 1977 from a United Kingdom medical school. Med Educ 1989;23:125-35

11 Firth-Cozens J. Source of stress in women junior house officers. BMJ 1990;301:89-91.

12 Bynoe G. Stress in women doctors. Br J Hosp Med 1994;51:267-8 Institute, 1994

14 Elliott FR. Professional and family conflicts in hospital medicine. Soc Sci Med 1979;13A:57-64

15 Allen I. Doctors and their careers. London: Policy Studies Institute, 1988

16 Tait A, Platt MJ. Women consultants: their background and trainingsome myths explored. Med Educ 1995;29:372-6.

17 Ritchie J, Spencer L. Qualitative data analysis for applied policy research. In: Bryman A, Burgess RG, eds. Analysing qualitative data. London:

18 NHS Management Executive. Junior doctors: the new deal. London: Department of Health, 1991.

19 Department of Health. A guide to specialist registrar training. Wetherby: DoH, 1996

(Accepted 22 February 2000)
13 Allen I. Doctors and their careers: a new generation. London: Policy Studies Routledge, 1994.

\section{Email submissions from outside the United Kingdom}

We are now offering an email submission service for authors from outside the UK. The address is papers@bmj.com

Ideally our email server would link seamlessly with our manuscript tracking system, but for now it does not, which is why we are offering the service only to authors outside the UK Most post in the UK arrives the next day, so UK authors have the least to gain in speed of delivery from email delivery. As soon as our systems improve we will invite email submissions from everyone.

If you choose to send your submission by email please would you send the text and any tables and figures as attached files, together with a covering letter giving all your contact details (postal address, phone, fax, and email address). We can read files created with most word processing, graphics, and spreadsheet programs.

When your submission is received in our email box you will receive an automatic acknowledgment to show that it has arrived. If the submission is incomplete we will contact you and ask you to resend the missing information.

Once the submission is complete we will register it on our manuscript tracking system and you will receive a standard acknowledgment in the post.

Letters to the editor should continue to be sent direct to bmj.com as rapid responsesor toletters@bmj.com 